\title{
Polar secretion of proteins via the Xcp type II secretion system in Pseudomonas aeruginosa
}

\author{
Freya Senf, Jan Tommassen and Margot Koster
}

\begin{abstract}
Correspondence
Margot Koster

M.C.Koster@uu.nl
\end{abstract}

Received 28 February 2008

Revised 21 May 2008

Accepted 18 June 2008

\author{
Department of Molecular Microbiology and Institute of Biomembranes, Utrecht University, $3584 \mathrm{CH}$ \\ Utrecht, The Netherlands
}

\begin{abstract}
The subcellular localization of the major type II secretion system of Pseudomonas aeruginosa, the Xcp system, was studied microscopically using a biarsenical ligand that becomes fluorescent upon binding to a tetracysteine motif (Lumio tag), which was fused to several Xcp components. Fusion of the Lumio tag to the $\mathrm{C}$ termini of the $X_{c p R}$ and $X_{c p S}$ proteins did not affect the functionality of these proteins. Fluorescence microscopy showed that they were predominantly localized to the poles of $P$. aeruginosa cells, when produced at levels comparable to chromosomally encoded XcpR and XcpS. In most labelled cells, the proteins were found at one of the poles, although bipolar localization was also observed. When produced in the absence of other Xcp components, labelled XcpS was still found to locate at the poles, whereas $X_{c p R}$ was evenly distributed in the cell. These data suggest that $X_{c p S}$, but not $\mathrm{XcpR}$, contains information required for polar localization. The polar location of the $X_{c p}$ machinery was further confirmed by the visualization of protease secretion with an intramolecularly quenched casein conjugate.
\end{abstract}

\section{INTRODUCTION}

Gram-negative bacteria have evolved several pathways for the secretion of proteins, one of them being the type II secretion pathway (T2SS), also known as the general secretory pathway (GSP) (Filloux, 2004). Type II secretion is a two-step process. The substrates, which include a large variety of toxins and enzymes, are first translocated over the inner membrane via either the Sec or the TAT system. In the periplasm, the folded proteins are recognized by the T2SS and secreted into the extracellular environment. T2SSs are complex machines consisting of 12-16 components, which are generically named Gsp, and Xcp in the case of the main T2SS in Pseudomonas aeruginosa. Many components of T2SSs share considerable sequence similarity with proteins of type IV pili and their assembly apparatus, the competence systems of Gram-positive bacteria, and components of the archaeal flagellum (Peabody et al., 2003), indicating an ancient evolutionary origin.

The Xcp system of $P$. aeruginosa is important for the secretion of many different proteins, including elastase, lipase, phospholipases, chitin-binding protein and exotoxin A. The system is encoded by $12 x c p$ genes (Filloux et al., 1998; Koster et al., 2000). Production of the system is regulated by quorum sensing (Chapon-Hervé et al., 1997; De Kievit \& Iglewski, 1999), and it has been estimated that

Abbreviations: GFP, green fluorescent protein; T2SS, type II secretion system.

A supplementary table listing the oligonucleotides used is available with the online version of this paper.
50-100 Xcp complexes are present in the cell at high cell densities (Brok et al., 1999). The secretion channel in the outer membrane is thought to be formed by the secretin XcpQ (GspD). The five components XcpT (GspG), XcpU (GspH), XcpV (GspI), XcpW (GspJ) and XcpX (GspK) share homology in their $\mathrm{N}$ termini to the subunits of type IV pili, and putatively assemble into a pseudopilus important for pushing the substrates through the secretion pore. These pseudopilins are produced with a leader peptide, which is cleaved off by the prepilin peptidase XcpA (GspO) (Bally et al., 1992; Bleves et al., 1998; Nunn \& Lory, 1992). The energy that is required for assembly of the pseudopilus and the extrusion of substrates is in all probability generated in the cytoplasm by the ATPase XcpR (GspE) (Camberg \& Sandkvist, 2005; Robien et al., 2003), which is part of the inner-membrane platform further consisting of the integral inner-membrane proteins XcpY (GspL), XcpZ (GspM) and XcpS (GspF) (Py et al., 2001; Robert et al., 2005b). XcpP (GspC) is thought to form a bridge between the secretin and the inner-membrane platform (Bleves et al., 1999; Gérard-Vincent et al., 2002; Robert et al., 2005a).

Besides the Xcp system, there is another T2SS in $P$. aeruginosa, named $\mathrm{Hxc}$, which is functional under phosphate limitation and involved in the secretion of the low-molecular-mass alkaline phosphatase LapA (Ball et al., 2002). In addition, $P$. aeruginosa produces type IV pili, which are important for adhesion to various materials and for twitching motility caused by alternating extensions and retractions of the pilus (Mattick, 2002). The three systems depend on the same prepilin peptidase (XcpA/PilD) for 
processing of their prepilin subunits (Ball et al., 2002; Bally et al., 1992; Nunn \& Lory, 1991, 1992). The type IV pili are located at the old pole of the cell (Weiss, 1971) and the ATPases important for retraction and extension of the pilus have been shown by using fluorescent fusion proteins to localize to the cell poles (Chiang et al., 2005). Since type IV pili and their assembly apparatus are located at the poles and show high sequence similarity to the components of T2SSs, one could expect that the T2SSs also localize at the poles. The localization of the Xcp system has not been investigated so far, but, by using real-time monitoring of green fluorescent protein (GFP) fusions and visualization of active protease secretion in single cells (Scott et al., 2001), the T2SS of Vibrio cholerae was indeed found to be located at the old pole of the cell. In contrast, the T2SS of Klebsiella oxytoca was shown, using a similar approach with GFP as a reporter, to be evenly distributed over the cell surface (Buddelmeijer et al., 2006). These different findings make it of interest to study the location of T2SSs in other bacteria. In this study, the location of the Xcp machinery of $P$. aeruginosa was determined by fluorescence microscopy using Xcp proteins tagged with a tetracysteine motif (Lumio tag), as well as by visualizing protease secretion using an intramolecularly quenched casein conjugate.

\section{METHODS}

Bacterial strains and growth conditions. The Escherichia coli and P. aeruginosa strains used in this study are listed in Table 1. E. coli Top $10 \mathrm{~F}^{\prime}$ was used for general cloning purposes. The bacteria were grown at $37{ }^{\circ} \mathrm{C}$ in a modified Luria-Bertani (LB) broth (Tommassen et al., 1983) solidified with $1.5 \%$ agar for plating. For the detection of elastase secretion on plates, bacteria were streaked on LB plates containing $0.5 \%(\mathrm{w} / \mathrm{v})$ elastin (Sigma). After 2 days incubation at $37{ }^{\circ} \mathrm{C}$, the plates were evaluated for the presence of haloes around the colonies. For plasmid maintenance, the following antibiotics were used: for E. coli, $50 \mu \mathrm{g}$ ampicillin $\mathrm{ml}^{-1}, 10 \mu \mathrm{g}$ gentamicin $\mathrm{ml}^{-1}$ and $10 \mu \mathrm{g}$ tetracycline $\mathrm{ml}^{-1}$; and for $P$. aeruginosa, $40 \mu \mathrm{g}$ gentamicin $\mathrm{ml}^{-1}$. Gene expression was induced with IPTG.
Plasmids and DNA manipulations. Plasmids used in this study are listed in Table 2. Recombinant DNA methods were essentially as described by Sambrook et al. (1989). Plasmids were introduced by the $\mathrm{CaCl}_{2}$ procedure into E. coli (Sambrook et al., 1989) or by electroporation into P. aeruginosa (Enderle \& Farwell, 1998). PCRs were performed with the proofreading enzyme Pwo DNA polymerase (Roche). The oligonucleotides used are listed in Supplementary Table S1 (available with the online version of this paper). The PCR products were cloned into the pCRII-TOPO vector according to the manufacturer's protocol (Invitrogen). All PCR products were sequenced. To obtain the $g f p-x c p S$ fusion, encoding GFP fused to the $\mathrm{N}$ terminus of XcpS, the following strategy was used. A PCR was performed with oligonucleotides GFP (N-term) FW and GFP (Nterm) Rev using pGFPmut3.1 as the template to obtain the $g f p$ gene, and one with the oligonucleotides XcpS (GFP) FW and XcpS (GFP) Rev on pAX24 as the template to obtain the $x c p S$ gene. Both PCR products were cloned into the PCRII-TOPO vector and subsequently excised from the resulting plasmids with the restriction enzymes indicated in Table S1. The DNA fragments were then triple-ligated into HindIII/XbaI-digested pYRC using T4 DNA ligase (Fermentas), resulting in pYRC-GFPS. A similar procedure was followed to obtain the chimeric gene encoding $\mathrm{XcpR}$ with GFP at the $\mathrm{C}$ terminus. The oligonucleotides GFP (C-term) FW and GFP (C-term) Rev were used to amplify $g f p$, and XcpR (GFP) FW and XcpR (GFP) Rev were used to obtain $x c p R$. The DNA fragments were cloned via pCRIITOPO into HindIII/XbaI-digested pYRC, resulting in pYRC-RGFP. To create the genes encoding the different Lumio-tagged Xcp components, PCRs were performed with the following oligonucleotides: for the gene encoding $\mathrm{N}$-terminally Lumio-tagged $\mathrm{XcpP}$, Lumio XcpP FW and Lumio XcpP Rev 2; for the gene encoding Cterminally Lumio-tagged XcpR, XcpR Lumio FW 2 and XcpR Lumio Rev; for the gene encoding C-terminally Lumio-tagged XcpS, XcpS Lumio FW and XcpS Lumio Rev and for the gene encoding Nterminally Lumio-tagged XcpS, Lumio XcpS FW and Lumio XcpS Rev 2. In all these PCRs plasmid pAX24 served as the template. The pilB gene was amplified using chromosomal DNA, released from cells of PAO1 by $10 \mathrm{~min}$ incubation at $94{ }^{\circ} \mathrm{C}$ in water, as the template and primers PilB FW and PilB Rev, introducing in the resulting gene product an $\mathrm{N}$-terminal polyhistidine tag and a $\mathrm{C}$ terminal Lumio tag. The PCR products were cloned into the pCRIITOPO vector and the $x c p$ or pilB genes were excised from the resulting constructs with HindIII and $\mathrm{XbaI}$ and ligated into the HindIII-XbaI-digested pYRC, rendering the pYRC plasmid derivatives listed in Table 2.

Table 1. E. coli and P. aeruginosa strains

\begin{tabular}{|c|c|c|}
\hline Strain & Relevant characteristics ${ }^{\star}$ & Source or reference \\
\hline \multicolumn{3}{|l|}{ E. coli } \\
\hline Top10F' & 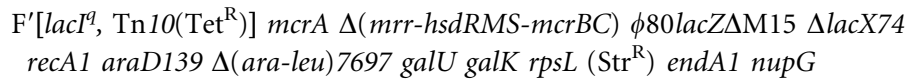 & Invitrogen \\
\hline \multicolumn{3}{|l|}{ P. aeruginosa } \\
\hline PAO1 & Wild-type & Holloway (1955) \\
\hline $\mathrm{PAO} 1 \Delta \mathrm{P}$ & $\mathrm{PAO} 1 \Delta x c p P$ & Bleves et al. (1999) \\
\hline PAN1 & $\mathrm{PAO} 25 x c p Q:: \mathrm{Gm}^{\mathrm{R}}$ & Bitter et al. (1998) \\
\hline PAN11 & PAO25 lasB:: $\mathrm{Km}^{\mathrm{R}} x c p R 54$ & Braun et al. (1998) \\
\hline $\mathrm{PAO} 1 \Delta \mathrm{S}$ & $\mathrm{PAO} 1 \Delta x c p S$ & Arts et al. (2007) \\
\hline DZQ40 & $\mathrm{PAO} 1 \Delta x c p P-Z$ & Ball et al. (1999) \\
\hline PAO25ME3 & PAO25 aprE:: $\Omega H g$ & Duong et al. (1994) \\
\hline PAN9 & PAO25ME3 lasB:: $\mathrm{Km}^{\mathrm{R}} x c p Q:: \mathrm{Gm}^{\mathrm{R}}$ & Braun et al. (1998) \\
\hline
\end{tabular}

${ }^{\star} \mathrm{Gm}$, Gentamicin; Km, kanamycin; Str, streptomycin; Tet, tetracycline. 
Table 2. Plasmids used

\begin{tabular}{|c|c|c|}
\hline Plasmid & Relevant characteristics ${ }^{\star}$ & Source or reference \\
\hline pCRII-TOPO & $\mathrm{Ap}^{\mathrm{R}} \mathrm{Km}^{\mathrm{R}}$; TOPO TA cloning vector & Invitrogen \\
\hline pGFPmut3.1 & $\mathrm{Ap}^{\mathrm{R}} ; g f p m u t 3.1$ & Clontech \\
\hline pYRC & $\mathrm{Gm}^{\mathrm{R}} ; \mathrm{pBBR} 1-\mathrm{MCS} 5 ;$ lacI; $\mathrm{P}_{\mathrm{lac}}$ & Arts et al. (2007) \\
\hline pYRC-RGFP & pYRC; $x c p R-g f p$ & This study \\
\hline pYRC-GFPS & pYRC; $g f p-x c p S$ & This study \\
\hline pYRC-RLum & pYRC derivative encoding C-terminally Lumio-tagged XcpR & This study \\
\hline pYRC-LumS & pYRC derivative encoding N-terminally Lumio-tagged XcpS & This study \\
\hline pYRC-SLum & pYRC derivative encoding C-terminally Lumio-tagged XcpS & This study \\
\hline pYRC-HisPilBLum & $\begin{array}{l}\text { pYRC derivative encoding N-terminally polyhistidine-tagged and } \\
\text { C-terminally Lumio-tagged PilB }\end{array}$ & This study \\
\hline
\end{tabular}

*Ap, Ampicillin; Gm, gentamicin; Km, kanamycin; Tet, tetracycline.

SDS-PAGE and immunoblot analysis. Bacterial cells were scraped from plates and suspended in PBS. The $\mathrm{OD}_{600}$ was measured and, from different cultures, similar amounts of cells were resuspended in SDS-PAGE sample buffer (2\% SDS, $5 \%$ DTT, $10 \%$ glycerol, $0.02 \%$ bromophenol blue, $0.1 \mathrm{M}$ Tris/ $\mathrm{HCl}, \mathrm{pH}$ 6.8). Extracellular proteins were precipitated from culture supernatants using $5 \%$ TCA, washed with acetone and resuspended in sample buffer. The amount of proteins loaded to the gels was equivalent to the quantity of protein present in the supernatant of $1 \mathrm{ml}$ of a culture with an $\mathrm{OD}_{600}$ of 0.1 . Samples were heated for $10 \mathrm{~min}$ at $95{ }^{\circ} \mathrm{C}$ and separated on SDSPAGE gels (Laemmli, 1970) with $0.2 \%$ SDS in the running gel. Proteins were stained with Coomassie brilliant blue or transferred to nitrocellulose membranes by Western blotting for immunodetection. The primary antisera used were anti-XcpP (Bleves et al., 1999), antiXcpR (see below), anti-XcpS (Arts et al., 2007), anti-GFP (Santa Cruz Biotechnology) and the monoclonal PentaHis antibody (Qiagen), all at $1: 1000$. Peroxidase-conjugated goat anti-rabbit or anti-mouse IgG antibodies (Biosource International) were used as secondary antibodies. Peroxidase activity was detected by chemiluminescence (Amersham). Anti-XcpR antiserum was raised in a rabbit immunized with a mixture of two synthetic peptides of XcpR (Sigma Genosys), namely [C]RKLMDETVRKPHGI and [C]PSIRDDGRRKVLE. These peptides were $\mathrm{N}$-terminally coupled to keyhole limpet haemocyanin. The rabbit was immunized at Sigma Genosys on day 0 with the conjugate containing $200 \mu \mathrm{g}$ of each of these peptides, and on days $14,28,42,56,70$ and 84 with $100 \mu \mathrm{g}$ each. The antiserum was collected 14 days after the final boost.

Fluorescence microscopy. For localization of Lumio-tagged proteins, cells were grown overnight at $37{ }^{\circ} \mathrm{C}$ on LB plates containing $1 \mu \mathrm{M}$ or $1 \mathrm{mM}$ IPTG. The cells were scraped from the plates and washed with $50 \mathrm{mM}$ HEPES ( $\mathrm{pH} 8$ ). Both FlAsH (Lumio Green, Invitrogen) (Griffin et al., 1998) and ReAsH (Lumio Red, Invitrogen) (Gaietta et al., 2002) ligands were tested. As the two labels revealed similar fluorescence patterns, but ReAsH showed brighter fluorescence than FlAsH, ReAsH was further used throughout this study. Samples $(100 \mu \mathrm{l})$ of cells at an $\mathrm{OD}_{600}$ of 0.1 in HEPES + buffer [50 mM HEPES pH 8, containing $4 \mu \mathrm{M}$ Lumio Red In-Cell Labelling Reagent (Invitrogen) and $200 \mu \mathrm{M}$ 1,2-ethanedithiol (Sigma-Aldrich)] were incubated for $3 \mathrm{~h}$ at $37{ }^{\circ} \mathrm{C}$ in the dark. Ethanedithiol was added to diminish unspecific binding of the Lumio reagents (Adams et al., 2002). The cells were then washed twice with $50 \mathrm{mM}$ HEPES $\mathrm{pH} 8$, and $10 \mu \mathrm{l}$ samples of the cells were placed on a glass slide and mounted by covering with a polylysine-coated coverslip. These coverslips were made in advance by dipping glass coverslips in a polylysine solution (Sigma), and drying them at room temperature. The polylysine fixed the cells to such an extent that microscopy was enabled without further need for fixation. The slides were used immediately. For protease secretion studies on single cells, cultures were grown in $\mathrm{LB}$ to an $\mathrm{OD}_{600}$ of at least 4 . The bacteria were harvested by centrifugation and washed twice with $0.9 \% \mathrm{NaCl}$. The cells were resuspended in spent culture medium of strain PAN9 as a source of autoinducers to an $\mathrm{OD}_{600}$ of 4 . A solution of $1 \%$ lowmelting-point agarose in $50 \mathrm{mM}$ HEPES pH 8 was cooled to $37{ }^{\circ} \mathrm{C}$. Bodipy FL Casein from the EnzChek Protease assay kit (Molecular Probes) was dissolved to a concentration of $20 \mu \mathrm{g} \mathrm{ml}^{-1}$ in $50 \mathrm{mM}$ HEPES buffer pH 8. A $2.5 \mu \mathrm{l}$ sample of this stock was added to $5 \mu \mathrm{l}$ of $20 \times$ Digestion buffer (Component B of the EnzChek Protease assay kit), $82.5 \mu \mathrm{l}$ of the agarose solution and $10 \mu \mathrm{l}$ of cell suspension. After quick mixing, $50 \mu \mathrm{l}$ of the suspension was placed on a slide and promptly topped with a coverslip. The slide was incubated for $30 \mathrm{~min}$ at $37^{\circ} \mathrm{C}$, after which it was immediately examined under the microscope. Using bright-field or fluorescent illumination, slides were viewed at $\times 1000$ magnification on an Axioskop microscope (Zeiss) fitted with a DFC420C camera (Leica). Exposure times were identical within the experiments. Leica Application Suite version 2.5.0 R1 software was used to collect the fluorescent and bright-field images. These images were processed with PhotoShop 7 (Adobe) using identical levels (brightness, contrast) for the same type of images.

\section{RESULTS}

\section{Construction and characterization of Lumio- tagged Xcp proteins}

To determine the cellular localization of the Xcp machinery, initially GFP was fused to the $\mathrm{N}$ terminus of $\mathrm{XcpS}$ and to the $\mathrm{C}$ terminus of XcpR. Production of these chimeric proteins in the $x c p S$ mutant PAO1 $\Delta S$ and the $x c p R$ mutant PAN11, respectively, did not restore the secretion of elastase, the major substrate of the Xcp system, as determined by the analysis of extracellular proteins by SDS-PAGE after growth in liquid medium (data not shown). These results indicated that these fusion proteins were not functional. Immunoblotting analysis showed that the intact fusion proteins were produced, but that 
breakdown products were also present in the cells (data not shown). Because of this instability and the lack of functionality, these GFP fusions were not suitable for localization studies.

Another labelling technique was therefore employed, which is based on the detection of proteins carrying a tetracysteine motif, named Lumio tag, with a biarsenical fluorophore. This ligand only becomes fluorescent upon covalent binding to the tetracysteine tag. The advantage over GFP fusions is that the small Lumio tag (six amino acids) is less likely to interfere with the functionality and the stability of the target proteins. Four different Lumiotagged Xcp proteins were engineered, namely XcpP with an N-terminal Lumio tag (LumXcpP), XcpR with a Cterminal Lumio tag (XcpRLum) and XcpS with either an N-terminal (LumXcpS) or a C-terminal Lumio tag (XcpSLum).

Production of C-terminally tagged XcpR and XcpS in the $x c p R$ and $x c p S$ mutant strains PAN11 and PAO1 $\Delta S$, respectively, restored the secretion of elastase, as was determined by analysis of extracellular protein profiles (Fig. 1a) and by evaluating the formation of haloes around colonies on elastin-containing plates (data not shown), showing that these tagged proteins are functional. In contrast, production of N-terminally tagged $\mathrm{XcpP}$ and $\mathrm{XcpS}$ in the $x c p P$ and $x c p S$ mutant strains $\mathrm{PAO} 1 \Delta \mathrm{P}$ and $\mathrm{PAO} 1 \Delta \mathrm{S}$, respectively, did not complement the secretion defect in either assay (shown for the extracellular protein profile in Fig. 1a). Moreover, the latter two proteins showed a strong dominant-negative effect in the wild-type strain PAO1, where their production interfered with elastase secretion in both assays (shown for the extracellular protein profile in Fig. 1b).

For the subcellular localization studies, it was important that the tagged proteins were produced at similar levels as the chromosomally encoded proteins in the wild-type strain in order to maintain the correct stoichiometry of the various components of the Xcp machinery. Induction of gene expression with different concentrations of IPTG revealed that the presence of $1 \mu \mathrm{M}$ IPTG resulted in the production of the recombinant C-terminally tagged XcpR (Fig. 1c) and XcpS (Fig. 1d) at levels comparable to those found for the chromosomally encoded XcpR and XcpS, respectively, in the wild-type strain PAO1. In contrast, higher concentrations of IPTG caused overproduction of the proteins (data not shown). Since fluorescence microscopy showed that $1 \mu \mathrm{M}$ IPTG was sufficient to induce production of the tagged protein in the majority of cells (see below), this concentration was used for the localization studies. Immunoblot analysis further confirmed that the production level of the Lumio-tagged protein XcpS was similar to that of plasmid-encoded wild-type XcpS (Fig. 1e), showing that the Lumio tag did not affect the stability of the protein. Moreover, the amounts of XcpSLum in the cells remained constant during labelling with the biarsenical fluorophore (data not shown).

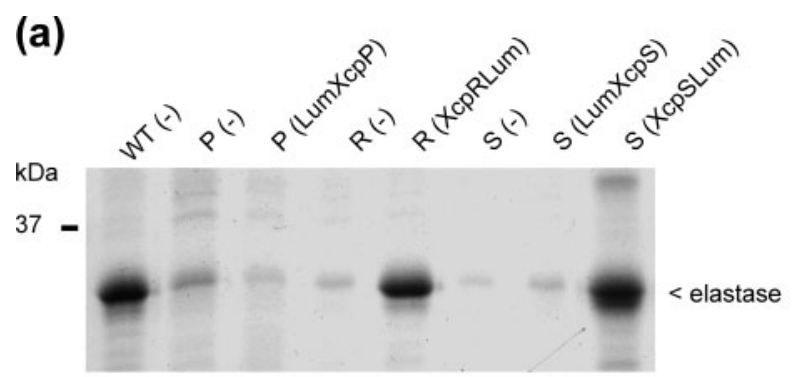

(b)
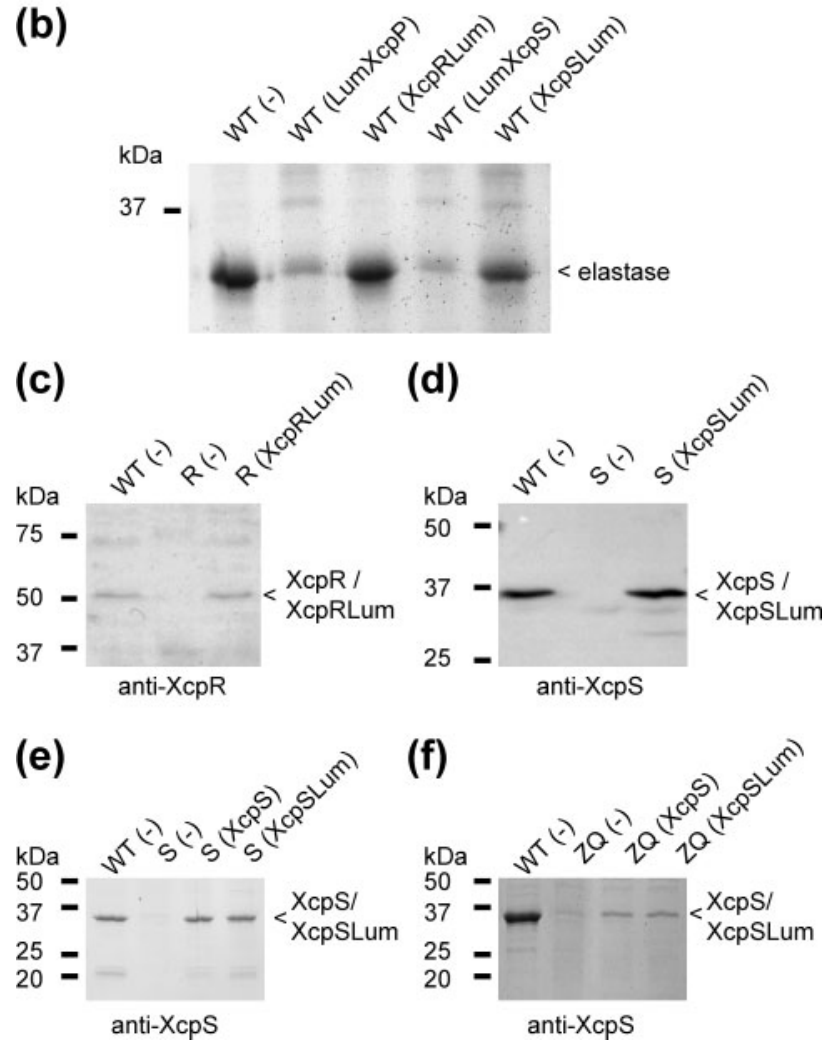

Fig. 1. Functionality and production of Lumio-tagged proteins. Shown are extracellular protein profiles $(a, b)$ and immunoblots of whole-cell lysates (c-f) of the wild-type strain PAO1 (WT) with the empty vector pYRC(-), pYRC-LumP encoding N-terminally Lumio-tagged XcpP (LumXcpP), pYRC-RLum encoding C-terminally Lumio-tagged $X_{c p R}\left(X_{c p R L u m}\right)$, pYRC-LumS encoding $\mathrm{N}$-terminally Lumio-tagged XcpS (LumXcpS) or pYRC-SLum encoding $\mathrm{XcpS}$ with a C-terminal Lumio tag (XcpSLum), the $x c p P$ mutant PAO1 $\Delta \mathrm{P}(\Delta \mathrm{P})$ with the empty vector or PYRC-LumP, the $x c p R$ mutant PAN11 $(\Delta \mathrm{R})$ with the empty vector or pYRCRLum and the $x c p S$ mutant PAO1 $\Delta S(\Delta S)$ with the empty vector, pYRC-LumS or pYRC-SLum, the DZQ40 strain lacking the entire $x c p Z-Q$ locus $(\Delta Z Q)$. $(a, b)$ Extracellular proteins of cells grown in liquid LB supplemented with $1 \mu \mathrm{M}$ (a) or $1 \mathrm{mM}$ (b) IPTG were precipitated with TCA, analysed by SDS-PAGE and stained with Coomassie brilliant blue. The position of the major Xcp substrate, elastase, is indicated. (c-f) Whole-cell preparations of bacteria grown on an LB plate containing $1 \mu \mathrm{M}(\mathrm{c}-\mathrm{e})$ or $1 \mathrm{mM}$ (f) IPTG were subjected to SDS-PAGE followed by transfer to nitrocellulose and immunodetection with the antisera indicated. The positions of the molecular mass marker proteins are indicated on the left. Only the relevant parts of the gels are shown. 


\section{Localization of Lumio-tagged proteins}

The functional recombinant proteins XcpRLum and XcpSLum were used to determine the localization of the Xcp system in P. aeruginosa. XcpSLum produced in the $x c p S$ mutant PAO1 $\Delta S$ localized predominantly at the cell poles (Fig. 2a). Depending on the experiment, between 50 and $80 \%$ of the cells were fluorescent and both unipolar and bipolar labelling was observed, although unipolar labelling was predominant: $\sim 80 \%$ of the labelled cells displayed unipolar fluorescence. Similarly, the $x c p R$ mutant PAN11 producing XcpRLum showed mainly unipolar fluorescence (Fig. 2b). As a control, a PilB derivative ( His $_{6}$ PilBLum) was constructed containing a C-terminal Lumio tag as well as an $\mathrm{N}$-terminal His tag (to enable immunodetection on Western blots). PilB is a homologue of XcpR involved in type IV pili biogenesis in P. aeruginosa, and its localization to both cell poles has been described (Chiang et al., 2005). Cells producing $\mathrm{His}_{6}$ PilBLum showed a very similar fluorescence pattern (Fig. 2f) as described previously for cells producing yellow-fluorescent protein fused to the PilB N terminus (Chiang et al., 2005), namely bipolar fluorescence in approximately $80 \%$ of the labelled cells. When any of the strains described above was incubated without the biarsenical reagent $\mathrm{ReAsH}$, no fluorescence was detectable, as shown for the cells producing C-terminally tagged XcpS in Fig. 2(g). Also no fluorescence was observed if ReAsh was added to cells of the wild-type strain not producing a Lumio-tagged protein (Fig. 2h). These results demonstrated that the fluorescence observed after incubation with $\mathrm{ReAsH}$ was generated by a specific interaction between the tetracysteine motif and the ligand.

When C-terminally tagged XcpS and XcpR were produced in the wild-type strain PAO1, the fluorescent signal was very diffuse and the polar patches were hardly visible, as shown for cells producing XcpSLum (Fig. 2e), which may

(a)

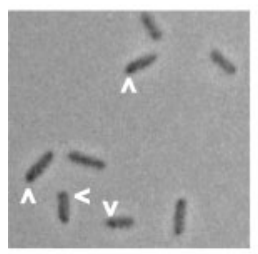

$\Delta \mathrm{S}$ (XcpSLum)

(c)
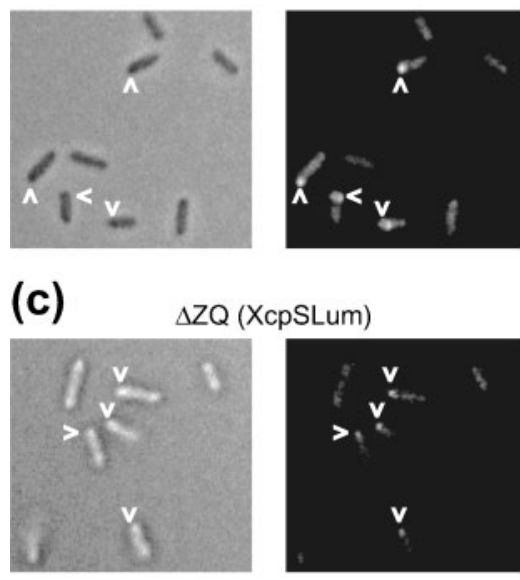

(e)

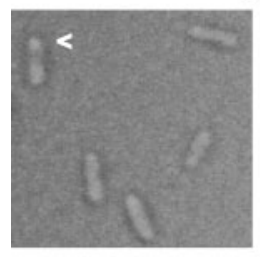

PAO1 (XcpSLum)

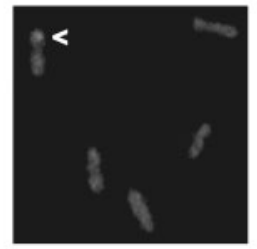

(g)

$$
\Delta \mathrm{S} \text { (XcpSLum) }
$$

- ReAsH

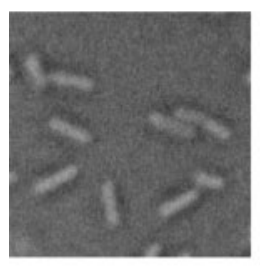

bright field

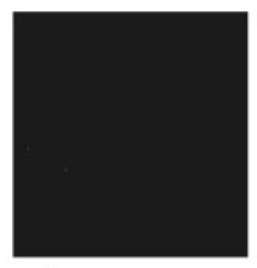

fluorescence (b)

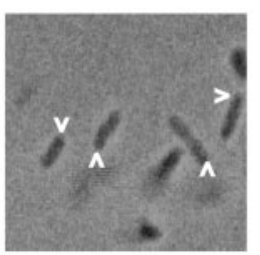

$\Delta \mathrm{R}$ (XcpRLum)

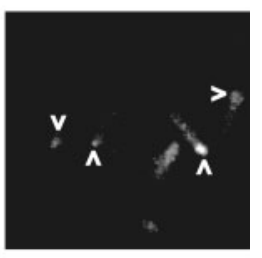

(d)
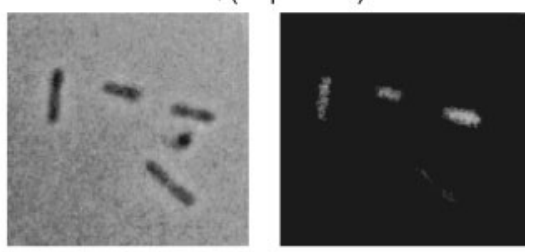

(f)
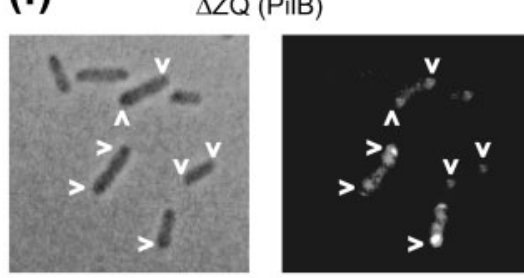

(h)

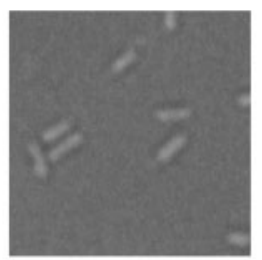

bright field
PAO1 (pYRC)

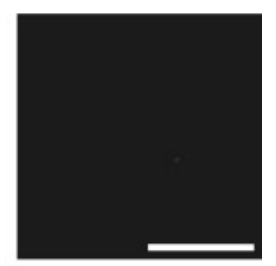

fluorescence
Fig. 2. Localization of Lumio-tagged proteins. (a) $x c p S$ mutant $(\Delta S)$ producing C-terminally Lumio-tagged XcpS (XcpSLum) from plasmid pYRC-SLum. (b) $x c p R$ mutant PAN11 $(\Delta \mathrm{R})$ producing C-terminally Lumio-tagged XcpR (XcpRLum) from plasmid pYRC-RLum. (c) $\Delta x c p Z-Q$ mutant $(\Delta Z \mathrm{Q})$ harbouring plasmid pYRC-SLum. (d) $\Delta Z Q$ harbouring the plasmid pYRC-RLum. (e) The wild-type strain PAO1 containing plasmid pYRC-SLum. (f) $\Delta Z \mathrm{Z}$ producing $\mathrm{N}$-terminally polyhistine-tagged and C-terminally Lumio-tagged PilB from plasmid pYRC-HisPilBLum. (g) $\Delta S$ harbouring plasmid pYRC-SLum. (h) PAO1 with the empty vector ( $p Y R C$ ). For all panels: cells were grown overnight on an LB plate containing $1 \mu \mathrm{M}(\mathrm{a}, \mathrm{b}, \mathrm{e}-\mathrm{h})$ or $1 \mathrm{mM}$ (c, d) IPTG. Cells scraped from the plate were suspended in HEPES buffer with $(a-f, h)$ or without $(g)$ the biarsenical ReAsH-reagent and incubated for $3 \mathrm{~h}$. Fluorescent images captured using a tetramethylrhodamine isothiocyanate filter are on the right, corresponding bright-field images on the left. Fluorescent poles are indicated with arrowheads. The scale bar represents $5 \mu \mathrm{m}$. 
be caused by competition between the recombinant proteins and the chromosomally encoded XcpS and XcpR for the polar location. Indeed, when C-terminally tagged $\mathrm{XcpR}$ or XcpS were overproduced in their respective mutant strains PAN11 and PAO1 $\Delta S$ by induction with $1 \mathrm{mM}$ IPTG, fluorescence also became more diffuse and the fluorescent foci, although still visible at the poles, became less clear (data not shown), indicating that excess $\mathrm{XcpR}$ and XcpS also occupy other cellular positions.

The recombinant proteins were also produced in strain DZQ40, which is deleted for the entire $x c p$ locus. No XcpSLum could be detected by immunoblotting in cells grown in the presence of $1 \mu \mathrm{M}$ IPTG, which is consistent with previous observations that XcpS is unstable in the absence of the other Xcp proteins (Arts et al., 2007). However, upon induction with $1 \mathrm{mM}$ IPTG, cells produced small amounts of XcpSLum that were detectable by immunoblot analysis (Fig. 1f). The production levels of XcpSLum were similar to those of plasmid-encoded wildtype XcpS produced in the same strain grown in the presence of $1 \mathrm{mM}$ IPTG (Fig. 1f). Production of Cterminally tagged XcpS in the DZQ40 strain still resulted in fluorescence at the cell poles (Fig. 2c). In contrast, production of the recombinant XcpR protein in the absence of the other Xcp components resulted in uniform fluorescence, of cells grown in the presence of IPTG at $1 \mathrm{mM}$ (Fig. 2d) or $1 \mu \mathrm{M}$ (data not shown). These results show that XcpR requires the presence of other Xcp components for polar localization, while XcpS is confined to the poles in the absence of the rest of the machinery.

\section{Elastase secretion at the poles}

To visualize the site of type II secretion, an intramolecularly quenched casein derivative was used, which upon proteolysis releases highly fluorescent peptides. This method allows for the detection of extracellular protease activity of single cells, as was previously demonstrated for the release of T2SS-dependent proteases by $V$. cholerae (Scott et al., 2001). In this experiment, cells were used of the aprE mutant strain PAO25ME3, which is defective in the secretion of the type I secretion system substrate alkaline protease. The cells were grown overnight and then washed to remove extracellular elastase. Since both the Xcp machinery and its substrates are regulated by quorum sensing (Chapon-Hervé et al., 1997; De Kievit \& Iglewski, 1999), the cells were mixed with spent medium of an aprE lasB $x c p Q$ triple mutant (PAN9), which should contain autoinducers but neither alkaline protease nor elastase or any other protease secreted via the Xcp machinery. After addition of the casein conjugate, the mixture was embedded in agarose to immobilize the cells. After $30 \mathrm{~min}$ incubation, the bacteria were examined by fluorescence microscopy. Fluorescence was observed for approximately $10 \%$ of the cells and occurred only at a single pole (Fig. 3a). After longer incubation times, the number of fluorescent cells increased, but also the fluorescent spots increased in size due to the diffusion of the extracellular elastase, making it more difficult to determine the exact location of secretion. When cells of an $x c p Q$ mutant (PAN1) were used, no fluorescent spots were detected (Fig. 3b). Thus, the observed fluorescence requires a functional Xcp system. From these experiments, it can be concluded that active secretion by the Xcp machinery is restricted to the poles of the cell.

\section{DISCUSSION}

In this study, specific labelling of tetracysteine-tagged Xcp components with biarsenical ligands was employed to determine the localization of the main T2SS in $P$. aeruginosa. Since these fluorophores are membrane per-

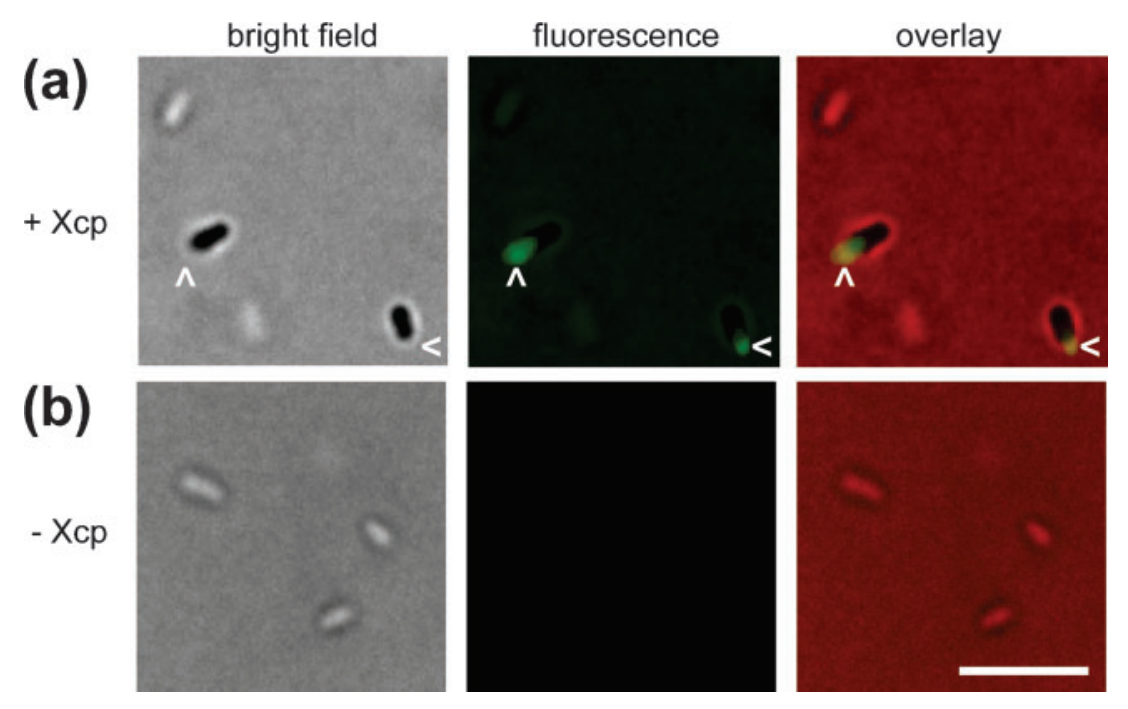

Fig. 3. Polar secretion of proteases by the $\mathrm{Xcp}$ system. Cells of the aprE mutant PAO25ME3 (+Xcp) (a) or the $x c p Q$ mutant PAN1 (-Xcp) (b) were resuspended in spent medium of the aprE las $B \times c p Q$ triple mutant PAN9 as a source of autoinducers and embedded in agarose containing Bodipy $\mathrm{FL}$ casein. After 30 min incubation, proteolysis of the substrate was evaluated. Arrowheads indicate fluorescence at the pole. Fluorescence images (FITC filter) are shown in the middle panels. The corresponding bright-field images are presented in the panels on the left. The false-coloured overlays of these images, created in Photoshop, are shown on the right, where green represents the FITC and red the bright-field image. The scale bar represents $5 \mu \mathrm{m}$. 
meable, this technique can be utilized to locate proteins in viable cells. In addition, the Lumio tag can be introduced in both cytoplasmic as well as periplasmic domains of a protein. Most importantly, this approach has the advantage that the tagging of a protein with a peptide of only six amino acid residues (CCPGCC) is less likely to interfere with function than its fusion to a fluorescent reporter protein such as GFP. Indeed, when the tag was fused to the $\mathrm{C}$ terminus of $\mathrm{XcpR}$, the resulting protein was fully functional, while GFP fused at the same position to XcpR affected the functionality.

$\mathrm{N}$-terminally tagged $\mathrm{XcpP}$ and $\mathrm{XcpS}$ were not functional and exerted a dominant-negative effect on secretion in the wild-type strain. The finding that a tag at the $\mathrm{N}$ terminus affected the functionality of XcpP was unexpected. XcpP is a bitopical inner-membrane protein, with a short cytoplasmic $\mathrm{N}$ terminus and a long periplasmic C-terminal region (Bleves et al., 1996), and a truncated derivative of the protein lacking the $\mathrm{N}$-terminal 17 amino acids residues has previously been shown to be functional (Bleves et al., 1999). It is therefore not clear why the presence of the tag blocks the function of XcpP, but it is possible that the tag, in spite of its small size, causes steric hindrance, thereby preventing interactions with other Xcp components.

The functional C-terminally Lumio-tagged XcpR and XcpS proteins were found at the poles of the cell, which is similar to the reported localization of T2SS (Eps) components of $V$. cholerae (Scott et al., 2001) but dissimilar to that of components of the Pul system of K. oxytoca (Buddelmeijer et al., 2006). The latter study showed that polar fluorescence can be an artefact caused by overproduction of the fluorescent protein. However, since the production levels of the tagged proteins were similar to that of chromosomally encoded XcpS and XcpR, the polar fluorescence in this study is unlikely to represent such an artefact. Moreover, it was also shown that secretion occurs at the poles by direct visualization of Xcp-mediated protease secretion. In this experiment, all the labelled cells exhibited unipolar secretion. The localization of Cterminally tagged XcpR and XcpS was predominantly unipolar, although bipolar fluorescence was observed as well. The Eps system of $V$. cholerae has been found to locate at the old cell pole, although upon maturation of the cells, a shift to a bipolar distribution was observed to take place (Scott et al., 2001). Such redistribution may occur also in $P$. aeruginosa.

When XcpS with a C-terminal Lumio tag was synthesized in the absence of all other Xcp components, the fluorescence, which was only observed when expression was induced with a high concentration of IPTG, was still polarly located. This result suggests that XcpS carries information to reach the poles. However, further studies are required to identify the targeting signal of XcpS as well as the polar component that recognizes this signal. When the tagged protein was produced in the wild-type strain in the presence of chromosomally encoded XcpS, the fluorescence became more diffuse, indicating that XcpSLum can occupy other cellular locations and competes with wild-type XcpS for a limited number of polar positions. The Lumio-tagged XcpR protein did not show polar localization in the absence of other Xcp proteins, suggesting that it is recruited to the pole by another protein. Ball et al. (1999) have shown that the XcpY protein is necessary and sufficient for the association of XcpR with the inner membrane, making $\mathrm{XcpY}$ the most likely candidate to recruit XcpR to the pole. Since XcpY and $\mathrm{XcpR}$ interact with XcpS (Robert et al., 2005b), the XcpS protein may be important to retain XcpY and XcpR to the pole. However, the instability of XcpS in the absence of other Xcp components (Arts et al., 2007) makes it unlikely that XcpS acts as a polar nucleation factor for the other Xcp components. GFP-EpsL, an XcpY homologue of V. cholera, requires EpsM, the XcpZ homologue, for polar localization, while GFP-EpsM protein localizes to the poles independent of the other components (Scott et al., 2001). The combined data suggest that several Xcp components may contain information required for polar assembly, while others are associated to the poles by binding to their interaction partners. A similar situation has been described for the VirB proteins of the type IV secretion system of Agrobacterium tumefaciens, where some components are independently targeted to the cell pole, whereas other components depend on other VirB proteins for polar localization (Judd et al., 2005).

The results of this study show that not only the biogenesis of type IV pili, but also the assembly of the main T2SS occurs at the cell poles of $P$. aeruginosa. The advantage of a polar localization of the T2SS is at this stage unknown. However, the restricted localization may facilitate the efficient assembly of these complex cell-envelope-spanning machines. This restricted localization does also raise many new questions. How do the components without localization signals reach their interaction partners? What is the signature in the proteins that restricts their localization to the pole and what are the cellular factors responsible for the recognition of this signal? Future research will focus on answering these questions.

\section{ACKNOWLEDGEMENTS}

We would like to acknowledge Cecile-Marie Boots for initial fluorescent microscopy studies and the students Phuong Ngo Yen and Walter van der Heijdt for their help with the construction of some of the plasmids used. This work was supported by the Research Council for Earth and Life Sciences (ALW) with financial aid from the Netherlands Organization for Scientific Research (NWO) (grant ALW 813-05-03).

\section{REFERENCES}

Adams, S. R., Campbell, R. E., Gross, L. A., Martin, B. R., Walkup, G. K., Yao, Y., Llopis, J. \& Tsien, R. Y. (2002). New biarsenical ligands and tetracysteine motifs for protein labeling in vitro and in vivo: synthesis and biological applications. J Am Chem Soc 124, 6063-6076. 
Arts, J., de Groot, A., Ball, G., Durand, E., El Khattabi, M., Filloux, A., Tommassen, J. \& Koster, M. (2007). Interaction domains in the Pseudomonas aeruginosa type II secretory apparatus component XcpS (GspF). Microbiology 153, 1582-1592.

Ball, G., Chapon-Hervé, V., Bleves, S., Michel, G. \& Bally, M. (1999). Assembly of XcpR in the cytoplasmic membrane is required for extracellular protein secretion in Pseudomonas aeruginosa. J Bacteriol 181, 382-388.

Ball, G., Durand, E., Lazdunski, A. \& Filloux, A. (2002). A novel type II secretion system in Pseudomonas aeruginosa. Mol Microbiol 43, 475-485.

Bally, M., Filloux, A., Akrim, M., Ball, G., Lazdunski, A. \& Tommassen, J. (1992). Protein secretion in Pseudomonas aeruginosa: characterization of seven $x c p$ genes and processing of secretory apparatus components by prepilin peptidase. Mol Microbiol 6, 1121-1131.

Bitter, W., Koster, M., Latijnhouwers, M., de Cock, H. \& Tommassen, J. (1998). Formation of oligomeric rings by XcpQ and PilQ, which are involved in protein transport across the outer membrane of Pseudomonas aeruginosa. Mol Microbiol 27, 209-219.

Bleves, S., Lazdunski, A. \& Filloux, A. (1996). Membrane topology of three Xcp proteins involved in exoprotein transport by Pseudomonas aeruginosa. J Bacteriol 178, 4297-4300.

Bleves, S., Voulhoux, R., Michel, G., Lazdunski, A., Tommassen, J. \& Filloux, A. (1998). The secretion apparatus of Pseudomonas aeruginosa: identification of a fifth pseudopilin, XcpX (GspK family). Mol Microbiol 27, 31-40.

Bleves, S., Gérard-Vincent, M., Lazdunski, A. \& Filloux, A. (1999). Structure-function analysis of XcpP, a component involved in general secretory pathway-dependent protein secretion in Pseudomonas aeruginosa. J Bacteriol 181, 4012-4019.

Braun, P., de Groot, A., Bitter, W. \& Tommassen, J. (1998). Secretion of elastinolytic enzymes and their propeptides by Pseudomonas aeruginosa. J Bacteriol 180, 3467-3469.

Brok, R., Van Gelder, P., Winterhalter, M., Ziese, U., Koster, A. J., de Cock, H., Koster, M., Tommassen, J. \& Bitter, W. (1999). The Cterminal domain of the Pseudomonas secretin XcpQ forms oligomeric rings with pore activity. J Mol Biol 294, 1169-1179.

Buddelmeijer, N., Francetic, O. \& Pugsley, A. P. (2006). Green fluorescent chimeras indicate nonpolar localization of pullulanase secreton components PulL and PulM. J Bacteriol 188, 2928-2935.

Camberg, J. L. \& Sandkvist, M. (2005). Molecular analysis of the Vibrio cholerae type II secretion ATPase EpsE. J Bacteriol 187, 249-256.

Chapon-Hervé, V., Akrim, M., Latifi, A., Williams, P., Lazdunski, A. \& Bally, M. (1997). Regulation of the Xcp secretion pathway by multiple quorum-sensing modulons in Pseudomonas aeruginosa. Mol Microbiol 24, 1169-1178.

Chiang, P., Habash, M. \& Burrows, L. L. (2005). Disparate subcellular localization patterns of Pseudomonas aeruginosa type IV pilus ATPases involved in twitching motility. J Bacteriol 187, 829-839.

De Kievit, T. R. \& Iglewski, B. H. (1999). Quorum sensing, gene expression, and Pseudomonas biofilms. Methods Enzymol 310, 117-128.

Duong, F., Soscia, C., Lazdunski, A. \& Murgier, M. (1994). The Pseudomonas fluorescens lipase has a C-terminal secretion signal and is secreted by a three-component bacterial ABC-exporter system. $\mathrm{Mol}$ Microbiol 11, 1117-1126.

Enderle, P. J. \& Farwell, M. A. (1998). Electroporation of freshly plated Escherichia coli and Pseudomonas aeruginosa cells. Biotechniques 25, 954-958.

Filloux, A. (2004). The underlying mechanisms of type II protein secretion. Biochim Biophys Acta 1694, 163-179.

Filloux, A., Bally, M., Murgier, M., Wretlind, B. \& Lazdunski, A. (1989). Cloning of $x c p$ genes located at the 55 min region of the chromosome and involved in protein secretion in Pseudomonas aeruginosa. Mol Microbiol 3, 261-265.

Filloux, A., Michel, G. \& Bally, M. (1998). GSP-dependent protein secretion in gram-negative bacteria: the Xcp system of Pseudomonas aeruginosa. FEMS Microbiol Rev 22, 177-198.

Gaietta, G., Deerinck, T. J., Adams, S. R., Bouwer, J., Tour, O., Laird, D. W., Sosinsky, G. E., Tsien, R. Y. \& Ellisman, M. H. (2002). Multicolor and electron microscopic imaging of connexin trafficking. Science 296, 503-507.

Gérard-Vincent, M., Robert, V., Ball, G., Bleves, S., Michel, G. P., Lazdunski, A. \& Filloux, A. (2002). Identification of XcpP domains that confer functionality and specificity to the Pseudomonas aeruginosa type II secretion apparatus. Mol Microbiol 44, 1651-1665.

Griffin, B. A., Adams, S. R. \& Tsien, R. Y. (1998). Specific covalent labeling of recombinant protein molecules inside live cells. Science 281, 269-272.

Holloway, B. W. (1955). Genetic recombination in Pseudomonas aeruginosa. J Gen Microbiol 13, 572-581.

Judd, P. K., Kumar, R. B. \& Das, A. (2005). Spatial location and requirements for the assembly of the Agrobacterium tumefaciens type IV secretion apparatus. Proc Natl Acad Sci U S A 102, 11498-11503.

Koster, M., Bitter, W. \& Tommassen, J. (2000). Protein secretion mechanisms in Gram-negative bacteria. Int J Med Microbiol 290, 325-331. Laemmli, U. K. (1970). Cleavage of structural proteins during the assembly of the head of bacteriophage T4. Nature 227, 680-685.

Mattick, J. S. (2002). Type IV pili and twitching motility. Annu Rev Microbiol 56, 289-314.

Nunn, D. N. \& Lory, S. (1991). Product of the Pseudomonas aeruginosa gene pilD is a prepilin leader peptidase. Proc Natl Acad Sci U S A 88, 3281-3285.

Nunn, D. N. \& Lory, S. (1992). Components of the protein-excretion apparatus of Pseudomonas aeruginosa are processed by the type IV prepilin peptidase. Proc Natl Acad Sci U S A 89, 47-51.

Peabody, C. R., Chung, Y. J., Yen, M. R., Vidal-Ingigliardi, D., Pugsley, A. P. \& Saier, M. H. (2003). Type II protein secretion and its relationship to bacterial type IV pili and archaeal flagella. Microbiology 149, 3051-3072.

Py, B., Loiseau, L. \& Barras, F. (2001). An inner membrane platform in the type II secretion machinery of Gram-negative bacteria. $E M B O$ Rep 2, 244-248.

Robert, V., Filloux, A. \& Michel, G. P. (2005a). Role of XcpP in the functionality of the Pseudomonas aeruginosa secreton. Res Microbiol 156, 880-886.

Robert, V., Filloux, A. \& Michel, G. P. (2005b). Subcomplexes from the Xcp secretion system of Pseudomonas aeruginosa. FEMS Microbiol Lett 252, 43-50.

Robien, M. A., Krumm, B. E., Sandkvist, M. \& Hol, W. G. (2003). Crystal structure of the extracellular protein secretion NTPase EpsE of Vibrio cholerae. J Mol Biol 333, 657-674.

Sambrook, J., Fritsch, E. F. \& Maniatis, T. (1989). Molecular Cloning: a Laboratory Manual, 2nd edn. Cold Spring Harbor, NY: Cold Spring Harbor Laboratory Press.

Scott, M. E., Dossani, Z. Y. \& Sandkvist, M. (2001). Directed polar secretion of protease from single cells of Vibrio cholerae via the type II secretion pathway. Proc Natl Acad Sci U S A 98, 13978-13983.

Tommassen, J., van Tol, H. \& Lugtenberg, B. (1983). The ultimate localization of an outer membrane protein of Escherichia coli K-12 is not determined by the signal sequence. EMBO J 2, 1275-1279.

Weiss, R. L. (1971). The structure and occurrence of pili (fimbriae) on Pseudomonas aeruginosa. J Gen Microbiol 67, 135-143.

Edited by: J. Anné 